\title{
Visualization of peptide-peptide interactions in FET biosensors with liquid-cell TEM
}

Li Xing, Ming-Siao Hsiao, Ahmad Islam, Nicholas Bedford, Rhett Martineau, Yen Ngo, Steve Kim, and Lawrence Drummy

Air Force Research Laboratory, Wright Patterson AFB OH 45433

Graphene-based field effect transistor (g-FET) sensors have been broadly applied in detection of biological macromolecules, such as RNA, DNA, peptides, and small toxic compounds [1]. The specificity and selectivity rely heavily on graphene-functionalization with biological recognizing elements (BREs) and the characterization of BRE engagement with target molecule is therefore one of the critical steps in sensor development. Transmission electron microscopy (TEM), because of its ability to offer high spatial resolution in compared to other image-based approaches, is a useful tool for examining the dynamic process involved in sensor detection. Here we present our TEM studies on a Neuropeptide Y specific g-FET biosensor in a liquid environment.

Neuropeptide Y (NPY) is one of the abundant proteins in brain [2], and it is involved in regulation of important biological and pathophysiological functions such as food uptake, energy homeostasis, circadian rhythm and cognition. NPY also serves as a major neurochemical component in stress response and a key element in modulation of emotional-affective behavior [3,4]. The level of NPY is therefore a potential biomarker of diagnosis for the disease condition related with dysregulation of NPYdependent physiological pathway(s). The non-invasive detection of NPY by g-FET sensor would play an important role in monitoring the human susceptibility to stressful or threat-related conditions.

To fabricate a sensor specific for NPY, we use a 12 amino acid NPY specific-binding peptide (N3 peptide) as a Biological Recognition Element that was identified using phage display. Chemical Vapor Deposited few-layer graphene was transferred to a liquid electrochemical TEM (Fig 1A) and the NPYN3 interaction was examined using an image-corrected FEI Titan microscope in the liquid cell. When fused with glutathione S-transferase (GST), the NPY full length protein assembles into a micelle-like structure of 20nm in solution containing three-copies of GST-NPY dimer, as it was determined with cryo-electron microscopy and single particle reconstruction (Fig 1c). GST-NPY micelles were able to freely move until binding onto graphene, confirming the existence of dynamic environment inside the liquid cell. Electron-water interaction were minimized using low-dose techniques, and not enough to induce significant $\mathrm{pH}$ change within the liquid cell [5]. The plot of GST-NPY trajectory showed that the micelle appeared to have localized vibration after binding to graphene (Fig 1b), a motion that is significantly different from the well-defined Brownian motion. The range of the micelle motion is on the order of the length of N3-peptide (Fig 1d), demonstrating that the GST-NPY micelle was tethered on graphene through the interaction with N3-peptide. Therefore, N3-peptide is a robust BRE that is able to induce NPY-detection on functionalization FET sensor and likely to be more robust than NPY antibodies.

References:

[1] Nehra, A. and Pal Singh, K., Biosens Bioelectron 2015, 74, 731.

[2] Sajdyk, T. J.; et al. Neuropeptides 2004, 38, 225.

[3] Reichmann, F. and Holzer, P. Neuropeptides 2016, 55, 99. 
[4] Tasan, R. O.; et al. Neuropeptides 2016, 55, 111.

[5] Schneider, N. M.; et al. J Phys Chem C 2014, 118, 22372.
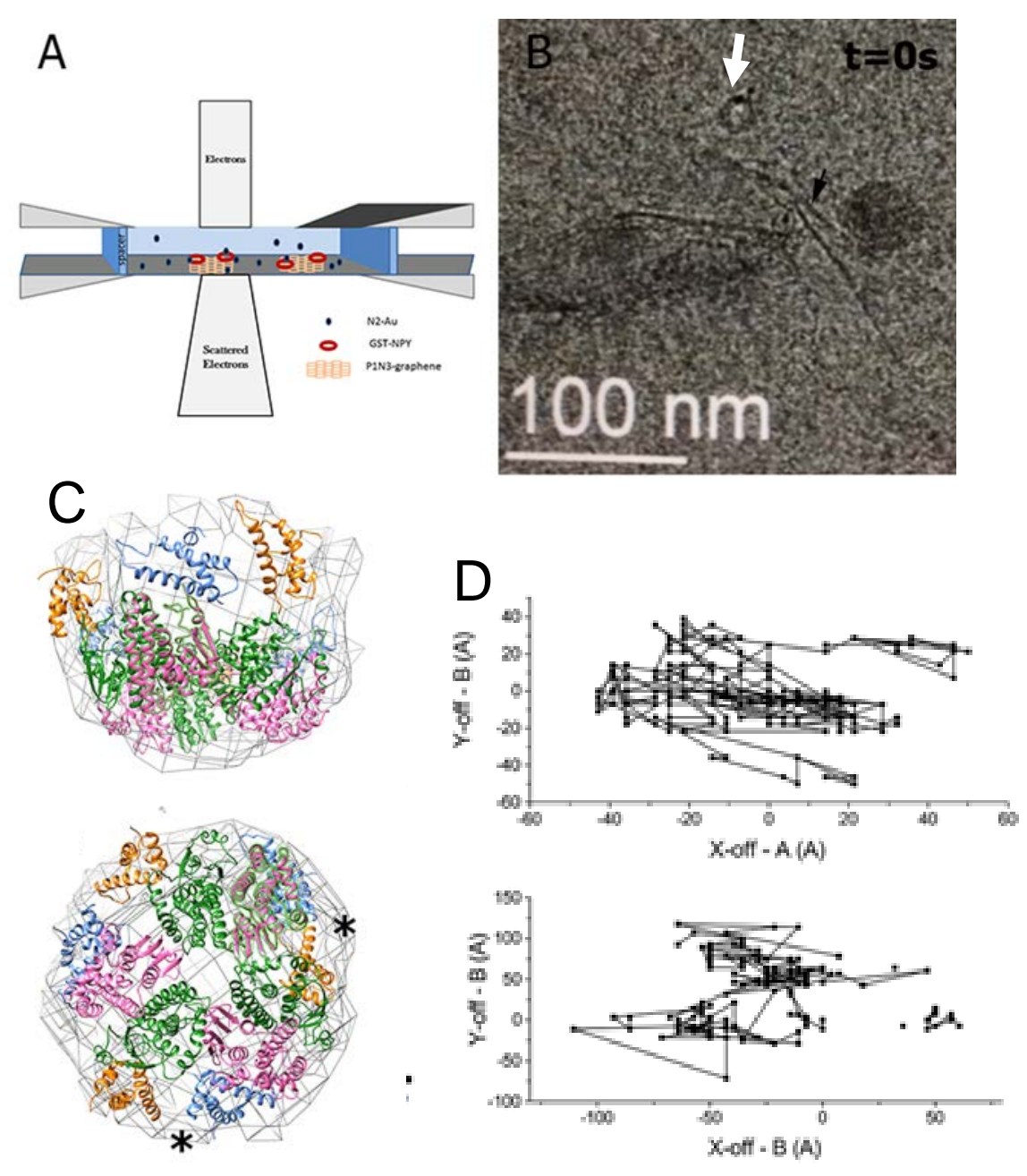

Figure 1. (A) The schematic illustration of graphene-based FET-sensor inside a liquid cell and the assembly of BRE with GST-NPY micelles under transmission electron microscope. (B) An image of GST-NPY binding with liquid cell from the video collected under low dose condition. The GST-NPY micelle (white arrow) observed in high contrast and mobile inside the liquid cell until it was tethered onto the graphene. (C) GST-NPY micelle appeared as cup-shaped density (grey mesh) that consists of three copies of dimeric NPY full length protein (gold- and cyan-colored ribbon structures), fused respectively on the C-terminal end of GST dimer (magenta- and green-colored ribbon structures). (D) The trajectory plots show respectively the local vibration GST-NPY micelles, in which the range of motion correlates to the length of BREs. 